Original paper

\title{
Incorporation of tumor motion directionality in margin recipe: The directional MidP strategy
}

\author{
Loïc Vander Veken ${ }^{\text {a, }}$, David Dechambre ${ }^{\mathrm{b}}$, Edmond Sterpin ${ }^{\mathrm{a}, \mathrm{c}}$, Kevin Souris ${ }^{\mathrm{a}}$, \\ Geneviève Van Ooteghem $^{\mathrm{a}, \mathrm{b}}$, John Aldo Lee ${ }^{\mathrm{a}}$, Xavier Geets ${ }^{\mathrm{a}, \mathrm{b}}$ \\ ${ }^{a}$ UCLouvain, Institut de Recherche Experimentale et Clinique (IREC), Center of Molecular Imaging, Radiotherapy and Oncology(MIRO), 1200 Brussels, Belgium \\ ${ }^{\mathrm{b}}$ Radiation Oncology Department, Cliniques Universitaires Saint-Luc, 1200 Brussels, Belgium \\ ${ }^{\mathrm{c}}$ KULeuven Department of Oncology, Laboratory of Experimental Radiotherapy, 3000 Leuven, Belgium
}

\section{A R T I C L E I N F O}

\section{Keywords:}

Tumor motion

PTV

Margin recipe

Mid Position

\begin{abstract}
A B S T R A C T
Purpose: Planning target volume (PTV) definition based on Mid-Position (Mid-P) strategy typically integrates breathing motion from tumor positions variances along the conventional axes of the DICOM coordinate system. Tumor motion directionality is thus neglected even though it is one of its stable characteristics in time. We therefore propose the directional MidP approach (MidP dir), which allows motion directionality to be incorporated into PTV margins. A second objective consists in assessing the ability of the proposed method to better take care of respiratory motion uncertainty.

Methods: 11 lung tumors from 10 patients with supra-centimetric motion were included. PTV were generated according to the MidP and MidP dir strategies starting from planning 4D CT.

Results: PTV ${ }_{\text {MidP dir volume didn't differ from the PTV }}$ MidP volume: $31351 \mathrm{~mm}^{3}$ IC95\% [17242-45459] vs. 31003 $\mathrm{mm}^{3}$ IC95\% [ 17347-44659], $\mathrm{p}=0.477$ respectively. PTV MidP dir morphology was different and appeared more oblong along the main motion axis. The relative difference between 3D and 4D doses was on average $1.09 \%, \mathrm{p}=$ 0.011 and $0.74 \%, p=0.032$ improved with directional MidP for $\mathrm{D}_{99 \%}$ and $\mathrm{D}_{95 \%}$. $\mathrm{D}_{2 \%}$ was not significantly different between both approaches. The improvement in dosimetric coverage fluctuated substantially from one lesion to another and was all the more important as motion showed a large amplitude, some obliquity with respect to conventional axes and small hysteresis.

Conclusions: Directional MidP method allows tumor motion to be taken into account more tightly as a geometrical uncertainty without increasing the irradiation volume.
\end{abstract}

\section{Introduction}

Breathing motion is well-known as a significant cause of altered dose delivery to target volume [1]. Radiation therapy of mobile tumors therefore requires dedicated methodologies. In contrast to sophisticated motion mitigation techniques based on the synchronization between beam delivery and instantaneous tumor location (e.g. gating or tracking), margin strategies can be easily implemented on conventional linear accelerators (linacs) without any addition of specialized device. This approach thus remains the most widespread in clinical routine [2].

Based on a four-dimensional computed tomography (4D-CT), two methods are commonly used to compute safety margins. The first involves the creation of an Internal Target Volume (ITV) encompassing all tumor positions in the respiratory cycle. To this volume is added a margin compensating for other geometrical uncertainties in order to generate the Planning Target Volume (PTV ${ }_{\text {ITV}}$ ) [3]. It has been shown, however, that PTV ITV is overconservative [4]. In accordance with the mathematical formalism established by van Herk et al [5], the second method, including both Mid-Position (MidP) and Mid-Ventilation (MidV) strategies, handles breathing motion as a random error that smears the dose distribution. In the Mid-V method, delineations are performed on the 4D CT phase closest to the time-weighted average of all tumor positions [6]. This frame of the 4D CT data set is however subject to motion-related artefacts degrading image quality. Moreover, a systematic error is introduced because of the hysteresis of tumor motion. A refinement of the concept has consequently been devised, the MidP method [7]: target volumes are contoured on the MidP-CT which represents the patient's anatomy at its time-weighted average position over

\footnotetext{
* Corresponding author at: Avenue Hippocrate 54, boîte B1.54.07, 1200 Woluwe-Saint-Lambert, Belgium.

E-mail address: loic.vanderveken@uclouvain.be (L. Vander Veken).
} 
the respiratory cycle. A single volumetric expansion is then performed to

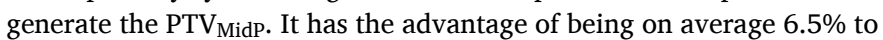
$13.9 \%$ smaller than PTV $\mathrm{PTV}_{\text {ITV }}$, thus lowering the probability of radiationinduced complications in surrounding healthy tissues [8-10].

It has been demonstrated that PTV $\mathrm{PidP}_{\mathrm{P}}$ ensures a satisfactory tumor dosimetric coverage [11-13]. Nevertheless, when the assumptions of the van Herk's probabilistic model are not respected, validity of computed margins is undermined, resulting in target under-dosage [14]. Among simplifications operated in the van Herk's margins model, tumor motion directionality is not considered. Indeed, only motion variances along the three conventional spatial dimensions are integrated in the calculation of geometrical uncertainties.

A concept of directional margin was firstly proposed by Alan McKenzie [15]. Briefly, his method involves successive dilations of the target volume with ellipsoids whose semi-diameters are aligned with the eigenvectors of the covariance matrices of systematic and random uncertainties. The length of their semi-diameters is equal to the square-root of the eigenvalue of its associated eigenvector. However, breathing motion is handled differently than the classical MidP approach. Actually, its contribution to the margin recipe is added linearly to other geometric errors combined in a quadratic way [16]. To our knowledge, no study has evaluated the potential benefit of a more refined description of the probability density function of tumor motion. The first goal of this work is therefore to propose a solution allowing integration of motion directionality in the MidP margin recipe. The second objective aims at quantifying the gain of our method in terms of tumor dosimetric coverage compared to the classical PTV $\mathrm{MidP}_{\mathrm{P}}$ approach.

\section{Methods}

\subsection{Definition of geometry}

Let $(x, y, z)$ be an orthogonal basis whose origin $(0,0,0)$ corresponds to the mid-position of CTV centers of mass $\left(\mathrm{CTV}_{\mathrm{CM}}\right)$ among all 4D CT phases. The $x, y$ and $z$ axes correspond to the left-to-right, antero-posterior and cranio-caudal directions, respectively.

\subsection{Consistent integration of motion directionality in margin recipe}

The $\mathrm{N}$ systematic errors are presumed to be independent of each other and the same applies to the relationship between their spatial components. The probability density function of geometrical uncertainties is supposed to be Gaussian. The first CTV extension compensating for systematic errors can thus be carried out in the same way as van Herk et al [5] by defining a confidence ellipsoid. This volume, called systematic PTV $\left(\right.$ PTV $\left._{s}\right)$, ensures CTV coverage for a given proportion of the patient population.

Margins $m$ can be calculated as follows in the orthogonal basis $(x, y, z)$ described in section 2.1:

$\left(\begin{array}{l}m_{P T V s}(x) \\ m_{P T V s}(y) \\ m_{P T V s}(z)\end{array}\right)=\alpha \cdot\left(\begin{array}{c}\sqrt{\sum_{i=1}^{n} \Sigma_{x}(i)^{2}} \\ \sqrt{\sum_{i=1}^{n} \Sigma_{y}(i)^{2}} \\ \sqrt{\sum_{i=1}^{n} \Sigma_{z}(i)^{2}}\end{array}\right)$

Coefficient $\alpha$ determines the percentage of patients whose CTV receives the minimal dose level that is allowed. $\Sigma(i)$ represents the standard deviation of a given systematic error.

Concerning random errors, preliminary assumptions are hypothesized to be identical as systematic one. In the classical model, perfect dose conformality is assumed for a spherical CTV of radius $|\vec{s}|$. Along direction defined by an arbitrary vector $\vec{r}$ quantifying systematic errors, the minimum dose level then happens at the point $\vec{r}+\vec{s}$ and can be calculated, as demonstrated by van Herk et al [5], by the convolution product between the probability density function of random errors and planned dose named $D_{\text {nominal }}$ :

$$
\begin{aligned}
D_{\text {blurred }}\{\vec{r}+\vec{s}\} & =D_{\text {nominal }} \cdot \int G\left(x, \sigma_{x}^{2}\right) \cdot G\left(y, \sigma_{y}^{2}\right) G\left(z, \sigma_{z}^{2}\right) S\{-\vec{x}-\vec{y} \\
& +\vec{r}+\vec{s}-\vec{z}, \vec{w}\} d x \cdot d y \cdot d z
\end{aligned}
$$

with $\mathrm{S}$, a step function whose vector $\vec{w}$ delimits an ellipsoid defining the volume of the $50 \%$ isodose:

$S(\vec{r}+\vec{s}, \vec{w})=\left\{\begin{array}{l}1, \text { for } \vec{r}+\vec{s} \leq \vec{w} \\ 0, \text { for } \vec{r}+\vec{s}>\vec{w}\end{array}\right.$

$G\left(x, \sigma_{x}^{2}\right), G\left(y, \sigma_{y}^{2}\right)$ and $G\left(z, \sigma_{z}^{2}\right)$ refer to the Gaussian probability density functions of combined random errors.

This approach leads, in clinical practice, to the computation of anisotropic margins compensating for random errors in the classical orthogonal basis regardless of the motion directionality. However, if tumor motion shows some obliquity, its spatial components cannot be treated as statistically independent. The mathematical corollary implies that the probability density function of combined random errors cannot be approximated by the product of its marginal distributions $G\left(x, \sigma_{x}^{2}\right)$, $G\left(y, \sigma_{y}^{2}\right)$ and $G\left(z, \sigma_{z}^{2}\right)$ due to non-null covariances between these dimensions. It is therefore necessary to decorrelate these components. Let $A_{t m}$ be the covariance matrix of tumor positions along its trajectory. It is calculated from the sample of $\mathrm{CTV}_{\mathrm{CM}}$ positions reconstructed by the $4 \mathrm{D}$ CT:

$\mathrm{A}_{t m}=\left(\begin{array}{ccc}\sigma_{x}(t m)^{2} & \operatorname{cov}_{x y}(t m) & \operatorname{cov}_{x z}(t m) \\ \operatorname{cov}_{x y}(t m) & \sigma_{y}(t m)^{2} & \operatorname{cov}_{y z}(t m) \\ \operatorname{cov}_{x z}(t m) & \operatorname{cov}_{y z}(t m) & \sigma_{z}(t m)^{2}\end{array}\right)$

The covariance matrix $\mathrm{A}_{\text {random }}$ resulting from the combination of the $\mathrm{K}$ independent random errors can therefore be written as follows:

$\mathrm{A}_{\text {random }}=\left(\begin{array}{ccc}\sum_{i=1}^{K} \sigma_{x}(i)^{2} & \sum_{i=1}^{K} \operatorname{cov}_{x y}(i) & \sum_{i=1}^{K} \operatorname{cov}_{x z}(i) \\ \sum_{i=1}^{K} \operatorname{cov}_{x y}(i) & \sum_{i=1}^{K} \sigma_{y}(i)^{2} & \sum_{i=1}^{K} \operatorname{cov}_{y z}(i) \\ \sum_{i=1}^{K} \operatorname{cov}_{x z}(i) & \sum_{i=1}^{K} \operatorname{cov}_{y z}(i) & \sum_{i=1}^{K} \sigma_{z}(i)^{2}\end{array}\right)$

Since tumor motion is the only random uncertainty considered as directional, we can simplify $\mathrm{A}_{\text {random }}$ as follows:

$\mathrm{A}_{\text {random }}=\left(\begin{array}{ccc}\sum_{i=1}^{K} \sigma_{x}(i)^{2} & \operatorname{cov}_{x y}(t m) & \operatorname{cov}_{x z}(t m) \\ \operatorname{cov}_{x y}(t m) & \sum_{i=1}^{K} \sigma_{y}(i)^{2} & \operatorname{cov}_{y z}(t m) \\ \operatorname{cov}_{x z}(t m) & \operatorname{cov}_{y z}(t m) & \sum_{i=1}^{K} \sigma_{z}(i)^{2}\end{array}\right)$

It must therefore be possible to define another orthogonal basis $(x$, $\left.y^{\prime}, z^{\prime}\right)$ in which $\mathrm{A}_{\text {random }}$ becomes diagonal. Such an orthogonal basis corresponds to the one defined by the eigenvectors of $\mathrm{A}_{\text {random }}$ :

$$
\begin{aligned}
\mathrm{A}_{\text {random }} & =\left(\begin{array}{ccc}
\sum_{i=1}^{K} \sigma_{x^{\prime}}(i)^{2} & \operatorname{cov}_{x^{\prime} y^{\prime}}(t m) & \operatorname{cov}_{x^{\prime} z^{\prime}}(t m) \\
\operatorname{cov}_{x^{\prime} y^{\prime}}(t m) & \sum_{i=1}^{K} \sigma_{y^{\prime}}(i)^{2} & \operatorname{cov}_{y^{\prime} z^{\prime}}(t m) \\
\operatorname{cov}_{x^{\prime} z^{\prime}}(t m) & \operatorname{cov}_{y^{\prime} z^{\prime}}(t m) & \sum_{i=1}^{K} \sigma_{z^{\prime}}(i)^{2}
\end{array}\right) \\
& =\left(\begin{array}{ccc}
\lambda_{x^{\prime}} & 0 & 0 \\
0 & \lambda_{y^{\prime}} & 0 \\
0 & 0 & \lambda_{z^{\prime}}
\end{array}\right)
\end{aligned}
$$

with $\lambda_{x^{\prime}}, \lambda_{y^{\prime}}, \lambda_{z^{\prime}}$ being the eigenvalues of the covariance matrix defined 
in $(x, y, z)$ associated with their corresponding eigenvector: $\overrightarrow{v_{x^{\prime}}}, \overrightarrow{v_{y^{\prime}}}$ and $\overrightarrow{v_{z^{\prime}}}$ respectively.

We can thus rewrite equation [2] as follows:

$$
\begin{gathered}
D_{\text {blurred }}\left\{\vec{r}+\vec{s}^{\prime}\right\}=D_{\text {nominal }} \cdot \int G\left(x^{\prime}, \sigma_{x^{\prime}}{ }^{2}\right) \cdot G\left(y^{\prime}, \sigma_{y^{\prime}}{ }^{2}\right) G\left(z^{\prime}, \sigma_{z^{\prime}}{ }^{2}\right) S\{-\vec{x}, \\
\left.-\vec{y},+\vec{r},+\vec{s},-\vec{z}, \overrightarrow{w^{\prime}}\right\} d x^{\prime} \cdot d y^{\prime} \cdot d z^{\prime}
\end{gathered}
$$

From equation [3], the operations and approximations are performed identically in Appendix 2 (equation B12 to B15) of van Herk et al [5]. We thus arrive at an identical margin recipe but in a different orthogonal basis:

$$
\left(\begin{array}{l}
m_{P \text { TVrandom }}\left(x^{\prime}\right) \\
m_{P \text { TVrandom }}\left(y^{\prime}\right) \\
m_{P \text { VVrandom }}\left(z^{\prime}\right)
\end{array}\right)=\beta \cdot\left(\begin{array}{c}
\sqrt{\lambda_{x^{\prime}}} \\
\sqrt{\lambda_{y^{\prime}}} \\
\sqrt{\lambda_{z^{\prime}}}
\end{array}\right)-\beta \cdot\left(\begin{array}{c}
\sigma p_{x^{\prime}} \\
\sigma p_{y^{\prime}} \\
\sigma p_{z^{\prime}}
\end{array}\right)
$$

Coefficient $\beta$ defines the minimum dose level delivered to the CTV.

As the penumbra of the dose distribution is in good approximation isotropic, the following equality applies as demonstrated in Appendix 1:

$$
\left(\begin{array}{c}
\sigma p_{x^{\prime}} \\
\sigma p_{y^{\prime}} \\
\sigma p_{z^{\prime}}
\end{array}\right)=\left(\begin{array}{c}
\sigma p_{x} \\
\sigma p_{y} \\
\sigma p_{z}
\end{array}\right)
$$

Ltd, UK). The same patient-specific audio-coaching was applied throughout treatment delivery. Lesions features are summarized in Table 1.

In order for breathing motion to have a non-negligible weight with respect to the other geometrical uncertainties, we have selected lesions with a supra-centimetric motion amplitude.

Lastly, as all tumors were treated with SBRT, no gross tumor volume (GTV) to CTV margin was added.

\subsection{Planning target volume definition}

\subsection{1. $P T V_{\text {MidP }}$}

For each patient, a MidP CT was reconstructed using a diffeomorfic Morphon algorithm implemented in the open-source Open-Reggui software [17]. Delineation of organs at risk (OARs) and GTV were performed in Raystation Planning system (clinical version 9B, RaySearch Laboratories, Stockholm, Sweden) on the MidP CT by the same radiation oncologist.

As shown in Fig. 1A for a simulated motion using the equation of

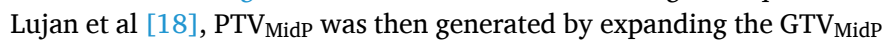
via safety margins calculated according to van Herk's formula as:

$M_{P T V_{M i d P}}=\alpha \cdot \sqrt{\Sigma_{T M}^{2}+\Sigma_{B L}^{2}+\Sigma_{S E T U P}^{2}+\Sigma_{D}^{2}}+\beta \cdot \sqrt{\sigma_{T M}^{2}+\sigma_{B L}^{2}+\sigma_{S E T U P}{ }^{2}+\sigma_{p}^{2}}-\beta . \sigma_{p}$

With

$\sigma p_{x}=\sigma p_{y}=\sigma p_{z}$

\subsection{Patients}

11 lung lesions from 10 patients treated by stereotactic body radiotherapy (SBRT) from 2018 to 2020 for stage I non-small cell lung carcinoma were included. A 4D-CT (Aquilion LB, Canon medical systems corporation, Japan) without contrast injection and retrospectively reconstructed in 10 temporally equivalent phases was performed. The slice thickness of the 4D-CT frames was set to $2 \mathrm{~mm}$. The planning CT was performed in supine position with hands above the head and with a vacuum bag as immobilization device. Abdominal compression (AC) was used in some cases depending on the instructions of the patient's physician. Patients underwent audio-coaching during CT acquisition based on their spontaneous respiratory rate and their external breathing signal was captured using the GateCT surface guided system (Vision RT where $\Sigma$ and $\sigma$ refer to the standard deviations of systematic and random errors, respectively. Labels "TM", "BL", "SETUP" and "D" correspond to tumor motion, baseline shift, set-up and delineation, respectively. Except for tumor motion, their values were taken from the literature and adapted to our institution protocols specificities [6,19-20]. Penumbra has been set at $5.5 \mathrm{~mm}$ using the same method as described by Wanet et al [14] for another treatment machine in our department. Briefly, it corresponds to the standard deviation of a cumulative Gaussian curve fitting the measured dose profile [21].

Regarding tumor motion, $\Sigma_{T M}$ corresponds to the residual error of the non-rigid co-registration algorithm and was equal to $0.5 \mathrm{~mm}$. $\sigma_{T M}$ coincides with the standard deviation of tumor motion. This parameter was individualized for each patient based on the standard deviation of the GTV center of mass coordinate set derived from all 4D CT phases.

Coefficient $\alpha$ was fixed at its conventional value of 2.5 ensuring that $90 \%$ of the patient population receives a given dose level. Coefficient $\beta$ was determined according to Thomas et al [12]: an overdosage was applied within the PTV so that the prescribed dose was at most equal to

\begin{tabular}{|c|c|c|c|c|c|c|c|c|c|c|}
\hline \multirow[t]{2}{*}{ Lesion } & \multirow[t]{2}{*}{ Location } & \multicolumn{3}{|c|}{$\begin{array}{l}\text { Tumor motion } \\
\text { amplitude (mm) }\end{array}$} & \multirow[t]{2}{*}{ Major motion axis (mm) } & \multirow[t]{2}{*}{ Hysteresis (mm) } & \multirow[t]{2}{*}{ Angle } & \multirow[t]{2}{*}{ GTV volume $\left(\mathrm{mm}^{3}\right)$} & \multirow[t]{2}{*}{ Linear coefficient } & \multirow[t]{2}{*}{ Prescription dose } \\
\hline & & LR & AP & CC & & & & & & \\
\hline 1 & LLL & 4.9 & 5.4 & 16.3 & 17.7 & 1.5 & $21.7^{\circ}$ & 17,100 & 0.95 & $4 \times 12 \mathrm{~Gy}$ \\
\hline 2 & RSL & 3.0 & 3.4 & 13.8 & 14.1 & 3.2 & $12.8^{\circ}$ & 2480 & 0.63 & $8 \times 7.5 \mathrm{~Gy}$ \\
\hline 3 & RIL & 3.6 & 3.7 & 18.2 & 18.3 & 4.6 & $7.0^{\circ}$ & 4500 & 0.50 & $4 \times 12 \mathrm{~Gy}$ \\
\hline 4 & LSL & 1.5 & 11.7 & 7.7 & 13.5 & 2.0 & $31.9^{\circ}$ & 842 & 0.90 & $4 \times 12 \mathrm{~Gy}$ \\
\hline 5 & LLL* & 2.3 & 7.8 & 22.4 & 22.4 & 8.0 & $9.8^{\circ}$ & 3359 & 0.53 & $3 \times 15 \mathrm{~Gy}$ \\
\hline 6 & RML & 3.9 & 8.9 & 7.6 & 12.4 & 2.6 & $37.7^{\circ}$ & 1287 & 0.97 & $3 \times 15 \mathrm{~Gy}$ \\
\hline 7 & RSL & 2.9 & 5.9 & 8.8 & 10.3 & 1.9 & $35.3^{\circ}$ & 20,724 & 0.96 & $5 \times 11 \mathrm{~Gy}$ \\
\hline 8 & RLL* & 4.1 & 7.1 & 21.0 & 22.6 & 1.8 & $20.8^{\circ}$ & 5210 & 0.98 & $8 \times 7.5 \mathrm{~Gy}$ \\
\hline 9 & LLL & 4.4 & 5.6 & 35.2 & 35.7 & 2.4 & $9.2^{\circ}$ & 7327 & 0.89 & $4 \times 12 \mathrm{~Gy}$ \\
\hline 10 & RLL & 3.7 & 8.2 & 26.0 & 27.1 & 8.1 & $13.2^{\circ}$ & 16,110 & 0.78 & $5 \times 10 \mathrm{~Gy}$ \\
\hline 11 & LLL & 3.0 & 8.8 & 16.7 & 18.8 & 3.9 & $26.0^{\circ}$ & 3307 & 0.92 & $5 \times 10 \mathrm{~Gy}$ \\
\hline
\end{tabular}

Table 1

Summary of lesions characteristics. Angle $=$ angle between major motion direction and conventional axis associated with the largest amplitude. Linear coefficient $=$ root mean square of linear correlation coefficient of tumor trajectory projected in the three spatial planes. * = Application of abdominal compression. 


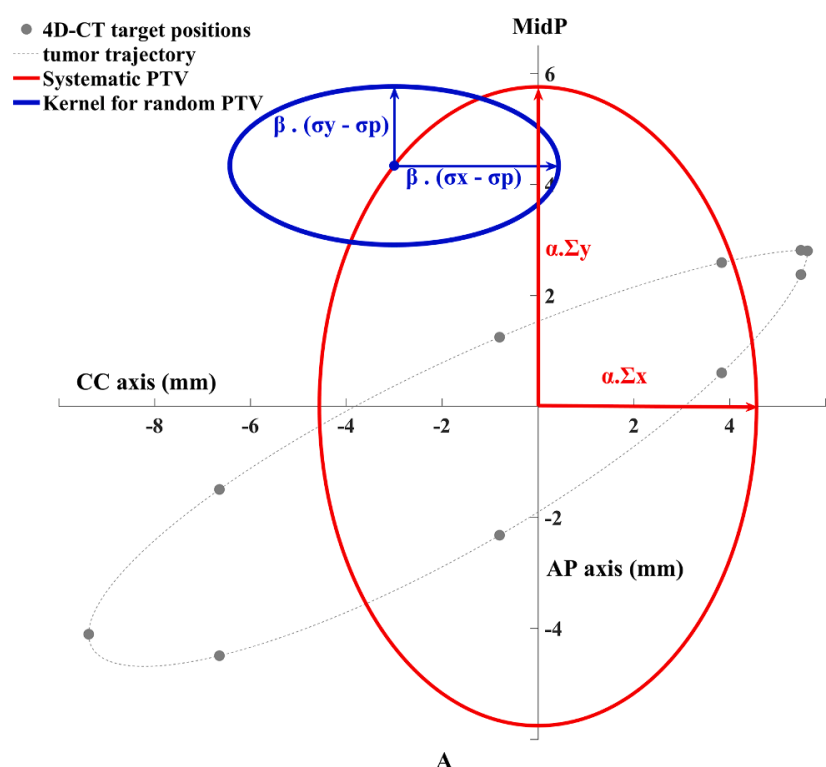

A

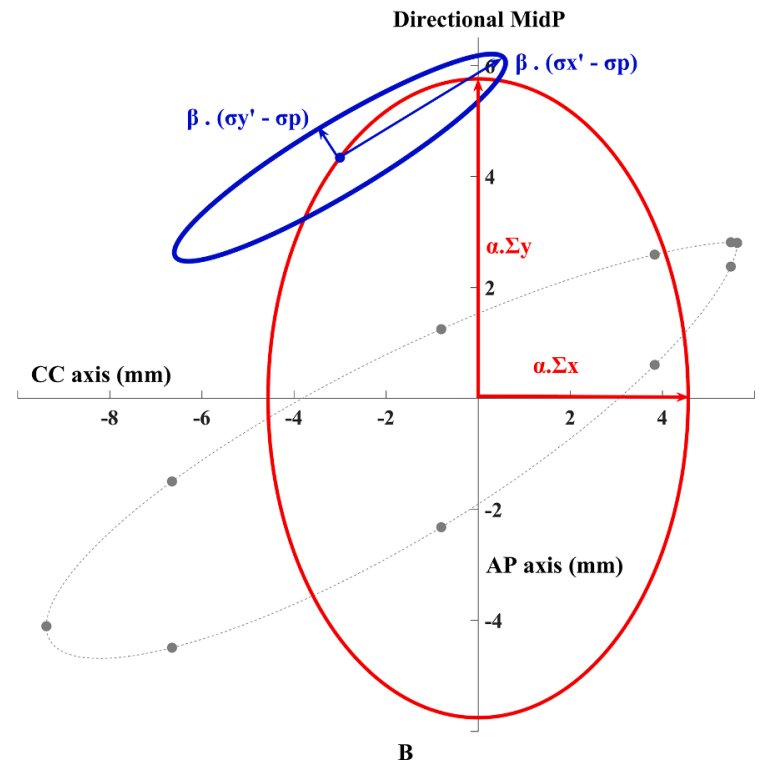

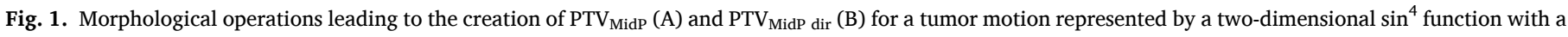

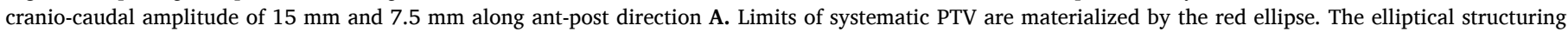

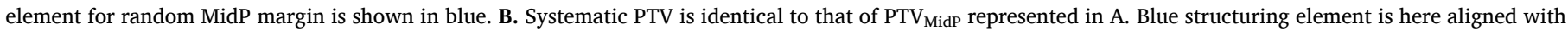

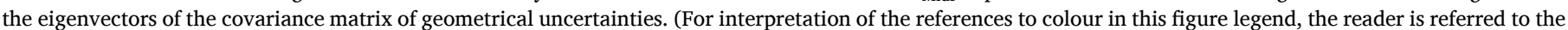
web version of this article.)

$90 \%$ of the maximum dose level. Coefficient $\beta$ equals 1.28 and guarantees that the GTV receives at least $90 \%$ of the prescribed dose in all planning scenarios.

\subsection{2. $P T V$ directional MidP}

The same MidP CT and GTV contours as for the PTV MidP were used. A first dilation of GTV $_{\text {MidP }}$ in the classical orthonormal axes was operated in order to generate the $\mathrm{PTV}_{\mathrm{s}}$. Margins were derived from the first square root of the van Herk's formula multiplied by the coefficient $\alpha$. This intermediate volume is identical between PTV $_{\text {MidP }}$ and PTV $_{\text {MidP dir. }}$

As shown in Fig. 1B, an expansion of $\mathrm{PTV}_{\mathrm{s}}$ was then performed using an ellipsoidal structuring element whose axes are aligned with the eigenvectors of $\mathrm{A}_{\text {random. }}$. The length of ellipsoid axes were equal to the eigenvalues associated with their respective eigenvectors to which the axes were parallel. This second volumetric extension leads to the crea-

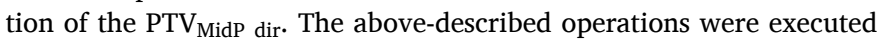
using a Matlab script developed in the Open Reggui software environment.

\subsection{Planning}

Treatments were planned by the same medical physicist in the treatment planning system Raystation (from Raysearch labs, version 9B) for a conventional linac (Infinity Elekta linac). Once optimized, treatment plans were computed on the average CT thanks to a convolution collapsed cone dose calculation algorithm. Voxel intensities of the average CT correspond to the mean of the 4D-CT phases, thus faithfully representing the average lung density during respiratory cycle [22]. The fractionation schedule was identical to the clinically validated one.

In accordance with ESTRO-ACROP and RTOG guidelines [21-23], planning objectives were the following: $\mathrm{D}_{99 \%}$ (dose received by $99 \%$ of the PTV volume) above $90 \%$ of the prescribed dose, $\mathrm{D}_{95 \%}$ equal to $100 \%$ of the prescription dose and $\mathrm{D}_{2} \%$ between 110 and $140 \%$ of the prescribed dose. Dose constraints to organs at risk were kept below literature recommendations [23-25].

Plans were compared using Paddick conformity index [26]:

CIPaddick $=\frac{T V_{I R}^{2}}{T V . I R}$ where $\mathrm{TV}_{\mathrm{IR}}$ corresponds to the PTV volume (TV) contained in the volume of the reference isodose (IR) [27] identified as the $D_{95 \%}$. We also reported Dice similarity index [28]:

$D S I=\frac{2 T V_{I R}}{T V+I R}$

\section{6. $4 D$-dose accumulation}

The delivered dose to the GTV, similar to Wanet et al [14], was determined by the accumulated dose to the extended GTV along its trajectory. It corresponds to the dilated GTV according to van Herk's formula for all geometrical uncertainties except tumor motion $\left(\Sigma_{T M}=\right.$ 0 and $\sigma_{T M}=0$ ). For this purpose, 3D dose maps were accumulated in the Mid Position using a hybrid method combining rigid and non-rigid methods. Dose accumulation workflow is illustrated in Fig. 2.

As the GTV is an anatomical structure, dose received by GTV $_{i}$ asso-

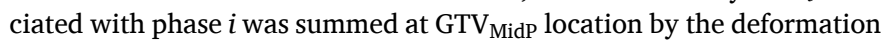
vector field $i\left(\mathrm{DVF}_{i}\right)$ obtained with the same deformable image registration (DIR) algorithm as to reconstruct corresponding MidP CT. Contribution of this distorted dose outside $\mathrm{GTV}_{\text {MidP }}$ was set to 0 . Since this accumulation critically depends on the DIR accuracy $[11,29-30]$, a correction was applied if centers of mass of $\mathrm{GTV}_{i}$ deformed by $\mathrm{DVF}_{i}$ and GTV $_{\text {MidP }}$ were not identical: a vector $c_{i}$ matching the center of mass of the deformed $\mathrm{GTV}_{i}$ to that of $\mathrm{GTV}_{\text {MidP }}$ was applied to the $\mathrm{DVF}_{i}$ deformed dose. It is known that lung tumors "track the dose" along their trajectory when type B dose engines are used for treatment planning [31]. Hence, 3D dose map has been particularized for each phase by recomputing the original plan on each 4D CT phase thanks to the Raystation's convolution collapsed cone algorithm.

As for the rest of the extended GTV volume, dose distribution was rigidly shifted by a translation vector $\mathrm{v}_{i}$ joining the center of mass of

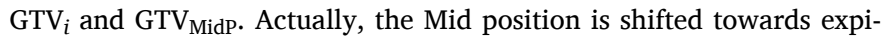
ratory phases and the selected tumors have a large motion amplitude. Highly mobile anatomy in the close environment of the tumor could consequently lead to an underestimation of the delivered dose to the extended $\mathrm{GTV}_{\mathrm{MidP}}$ with an exclusively non-rigid 4D dose accumulation. Indeed, substantial distension of lung tissue during respiratory cycle 


\section{Non rigid dose accumulation}

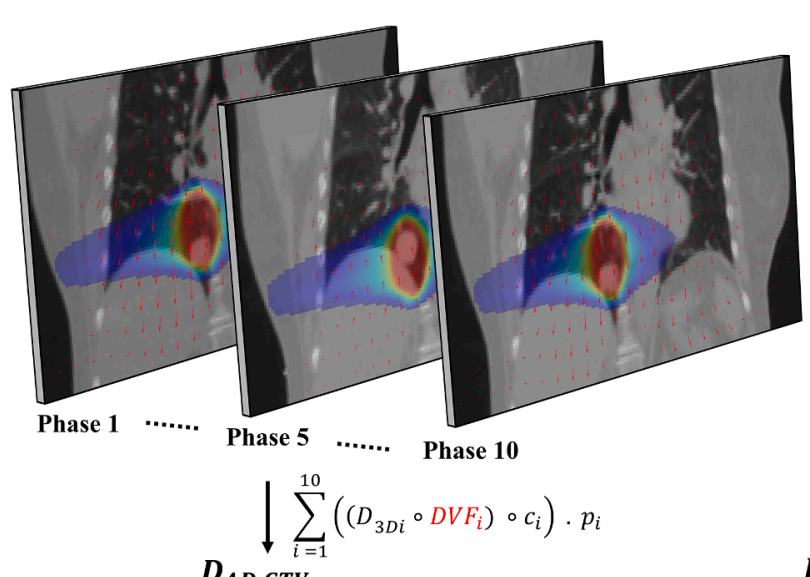

\section{Rigid dose accumulation}

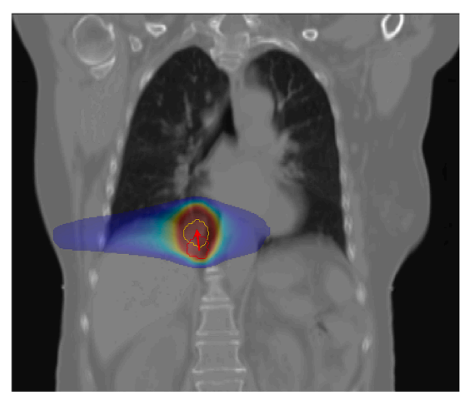

Average CT

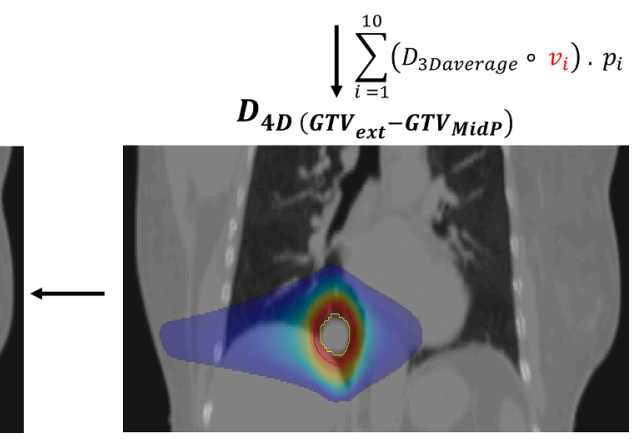

MidP CT

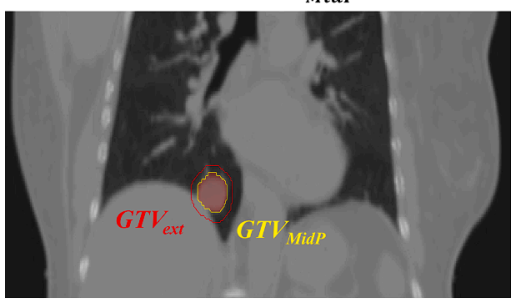

MidP CT
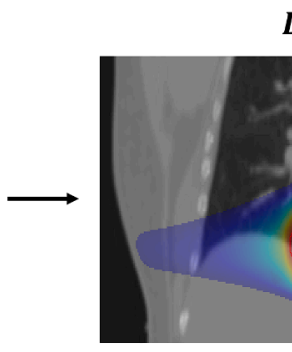

$D_{4 D G T V_{e x t}}$

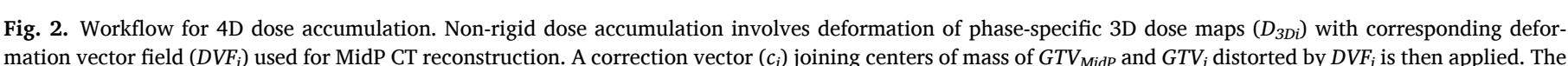

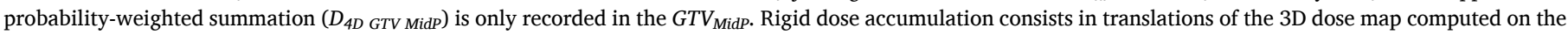

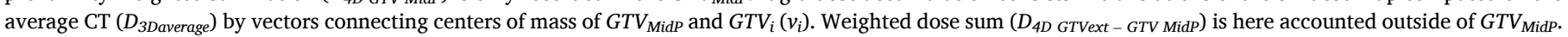
Final dose distribution $\left(D_{4 D}\right.$ GTVext $)$ is obtained by the addition of $D_{4 D}$ GTV MidP and $D_{4 D}$ GTVext - GTV MidP .

may result in the accumulation of dose voxels located outside the extended $\mathrm{GTV}_{i}$ within the extended $\mathrm{GTV}_{\text {Midp. The extended GTV being a }}$ purely geometrical volume, this kind of accumulation has no physical meaning. Contribution of this rigidly translated dose within GTV $_{\text {MidP }}$ was nullified. Finally, the distorted dose has here not been individualized on each phase and corresponds to the original one computed on the average CT. Density of its voxels is indeed closer to that of the GTV than on a single 4D CT phase and thus offers an acceptable trade-off for 4D dose accumulation [32].

The final 4D delivered dose is the sum of both accumulations.

Mathematically, the latter could be written as follows:

$D_{4 D G T V_{\text {ext }}}=D_{4 D G T V_{\text {MidP }}}+D_{4 D\left(G T V_{\text {ext }}-G T V_{\text {MidP }}\right)}$

with

$D_{4 D G T V_{\text {MidP }}}=\sum_{i=1}^{N}\left(\left(D_{3 D i}{ }^{\circ} D V F_{i}\right)^{\circ} c_{i}\right) p_{i}$

$D_{4 D\left(G T V_{\text {ext }}-G T V_{\text {MidP }}\right)}=\sum_{i=1}^{N}\left(D_{3 \text { Daverage }}{ }^{\circ} v_{i}\right) p_{i}$

where $\mathrm{p}_{i}$ corresponds to the tumor presence probability in phase $i$. Given the $4 \mathrm{D}$ CT reconstruction in 10 temporally equivalent phases, all $\mathrm{p}_{i}$ are uniformly equal to 0.1 .

Loss of dosimetric coverage induced by tumor motion was calculated by the relative differences between the $\mathrm{D}_{\mathrm{x} \%}$ (minimum dose received by $\mathrm{x} \%$ of the volume of interest) planned to the PTV and that delivered to the extended GTV. These differences were reported for the $\mathrm{D}_{99 \%}, \mathrm{D}_{95 \%}$ and $\mathrm{D}_{2 \%}$ and were noted $\Delta \mathrm{D}_{99 \%}, \Delta \mathrm{D}_{95 \%}$ and $\Delta \mathrm{D}_{2 \%}$, respectively. The absolute difference of $\Delta \mathrm{Dx} \%$ between MidP and directional MidP method corresponded to the final metric quantifying the difference in tumor dosimetric coverage between the two approaches.

\subsection{Statical analysis}

Statistical analyses were performed on IBM SPSS Statistics 26 software. The means of continuous variables were compared using twotailed and paired t-tests when data sample were normally distributed or Wilcoxon tests in case of non-Gaussian distributions. The significance threshold was set at $5 \%$.

\section{Results}

\subsection{Motion features}

As shown in Table 1, major motion axis amplitude ranges from 10.26 $\mathrm{mm}$ to $35.74 \mathrm{~mm}$. Lesions located in the upper or middle lobe show the smallest amplitudes. Two tumors ( ${ }^{\circ} 5$ and 10) feature a significant hysteresis of more than $8 \mathrm{~mm}$.

Angles reported in Table 1 correspond to the deviation between conventional axis related to the largest motion amplitude and major motion axis direction defined by the eigenvector associated with the highest eigenvalue. There seems to be an inverse relationship between motion angulation and its amplitude. Linear coefficient refers to the root mean square of the linear correlation coefficients (LCC) of the projected tumor trajectory in the 3 spatial planes $x y, x z$ and $y z$. A coefficient equal to 1 would mean a purely linear tumor motion morphology without any hysteresis. As can be observed in Table 1, hysteresis amplitude decreases with the linear coefficient.

\subsection{Margin and PTV volume}

As seen in Table 2, the directional MidP margin associated with the first principal component is systematically higher than the largest MidP margin. In contrast, the margin related to the third principal component 
Table 2

PTV random margins and volumes for MidP and directional MidP methods. Volume ratio refers to PTV MidPdir $_{\text {volume divided by PTV }}$ MidP volume.

\begin{tabular}{|c|c|c|c|c|c|c|c|c|c|}
\hline \multirow[t]{2}{*}{ Lesion } & \multicolumn{3}{|c|}{ Random margins - MidP (mm) } & \multirow[t]{2}{*}{$\operatorname{PTV}_{\text {MidP }}\left(\mathrm{mm}^{3}\right)$} & \multicolumn{3}{|c|}{ Random margins -MidP ${ }_{\text {dir }}(\mathrm{mm})$} & \multirow[t]{2}{*}{$\operatorname{PTV}_{\text {MidPdir }}\left(\mathrm{mm}^{3}\right)$} & \multirow[t]{2}{*}{ Volume ratio } \\
\hline & LR & AP & CC & & $\mathrm{X}^{\prime}$ & $Y^{\prime}$ & Z' & & \\
\hline 1 & 0.7 & 0.9 & 3.8 & 54,080 & 0.4 & 0.6 & 4.3 & 54,970 & 1.016 \\
\hline 2 & 0.6 & 0.7 & 3.1 & 13,950 & 0.4 & 0.6 & 3.3 & 13,460 & 0.965 \\
\hline 3 & 0.5 & 0.8 & 4.5 & 24,350 & 0.4 & 0.8 & 4.6 & 23,710 & 0.974 \\
\hline 4 & 0.4 & 2.2 & 1.1 & 6900 & 0.3 & 0.5 & 2.8 & 6390 & 0.926 \\
\hline 5 & 0.4 & 1.5 & 5.4 & 20,065 & 0.4 & 1.4 & 5.5 & 20,332 & 1.013 \\
\hline 6 & 0.6 & 2.0 & 1.2 & 9269 & 0.3 & 0.5 & 2.8 & 8907 & 0.961 \\
\hline 7 & 0.5 & 1.1 & 1.6 & 59,786 & 0.3 & 0.6 & 2.2 & 59,582 & 0.997 \\
\hline 8 & 0.6 & 1.3 & 5.6 & 25,820 & 0.4 & 0.6 & 6.2 & 26,650 & 1.032 \\
\hline 9 & 0.6 & 1.0 & 11.7 & 48,594 & 0.4 & 0.7 & 11.9 & 50,640 & 1.042 \\
\hline 10 & 0.5 & 1.5 & 8.1 & 59,047 & 0.3 & 1.2 & 8.4 & 61,097 & 1.035 \\
\hline 11 & 0.5 & 1.7 & 4.5 & 19,171 & 0.4 & 0.7 & 5.2 & 19,119 & 0.997 \\
\hline
\end{tabular}
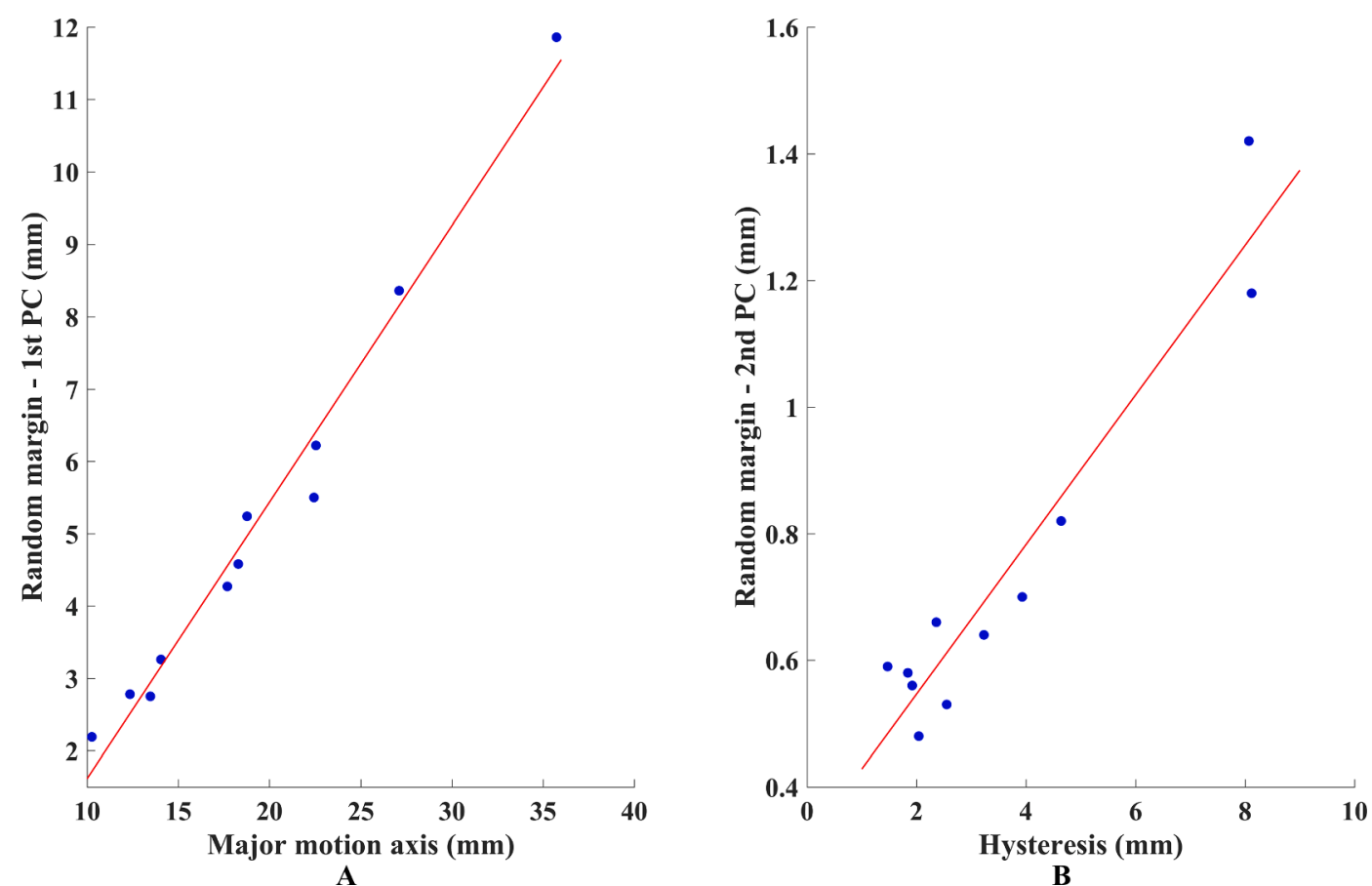

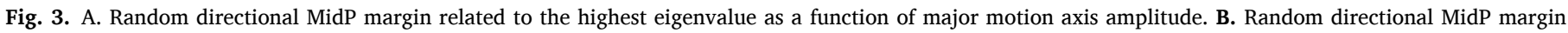
related to the intermediate eigenvalue as a function of hysteresis.

is always smaller than the smallest MidP margin. Graphs in Fig 3 show a clear proportional relationship between margin of the first principal component and major motion axis amplitude. The same trend is observed between the hysteresis amplitude and the margin associated with the second principal component.

From a volumetric point of view, the average PTV $\mathrm{V}_{\text {MidP dir volume did }}$

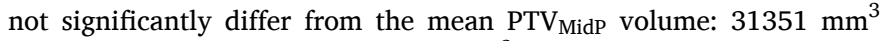
IC95\% [17242 - 45459] vs. $31003 \mathrm{~mm}^{3}$ IC95\% [ 17347-44659], $\mathrm{p}=$ 0,477 . However, as shown in Fig 4a, their spatial distribution appears not identical: PTV $\mathrm{V}_{\text {MidP dir }}$ is wider than $\mathrm{PTV}_{\text {MidP }}$ along the main motion axis but narrower along perpendicular directions.

\subsection{GTV dosimetric coverage}

Plans' conformity indices for both approaches were not significantly different. DSI index was 0.962 CI95\% [0.955-0.968] vs. 0.957 CI95\% [0.951-0.963], $\mathrm{p}=0.067$ for MidP and MidP ${ }_{\text {dir }}$ respectively. Paddick's conformity index was 0.925 CI95\% [0.912-0.938] vs. 0.916 CI95\% [0.905-0.928], $\mathrm{p}=0.063$ for MidP and MidP dir $_{\text {respectively. }}$.

Directional MidP significantly improved dose coverage for $\mathrm{D}_{99 \%}$ (mean difference of 1.087\% CI95\% [0.346-1.858], $\mathrm{p}=0.011$ ) and $\mathrm{D}_{95 \%}$ (average difference of $0.739 \%$ CI95\% [0.297-1.401], $\mathrm{p}=0.032$ ). The improvement of $\mathrm{D}_{99 \%}$ and $\mathrm{D}_{95 \%}$, as shown in Fig. 5, is at first approximation driven by the major motion axis amplitude. This correlation is however subject to significant variability. By contrast, the $\mathrm{D}_{2 \%}$ does not differ between the two techniques (average difference of 0.104 [-0.145-0.316], $\mathrm{p}=0.429)$. The results for $\Delta \mathrm{D}_{99 \%}, \mathrm{D}_{95 \%}$ and $\mathrm{D}_{2 \%}$ are compiled in Table 3.

\section{Discussion}

Compared to PTV MidP, PTV MidPdir shows a slight but statistically significant trend towards improved $\mathrm{D}_{99 \%}$ and $\mathrm{D}_{95 \%}$ without any increase of the irradiation volume. $\mathrm{D}_{2 \%}$ is not differently affected between both approaches. Higher tumor dosimetric coverage is particularly desirable as it allows potentially for a better local control [33-34]. In the context of SBRT, it has also been shown that the minimal 4D accumulated dose to the tumor could be a determinant of local failure [35]. However, the dose-response relationship at such high dose levels and in stereotactic conditions remains somewhat unclear [36-38]. Translation of the study results into clinical significance is thus not well established.

Beyond statistical analyses, it worth mentioning that the benefit of the directional MidP method is highly fluctuating from one lesion to another. Several determinants could theoretically explain this 


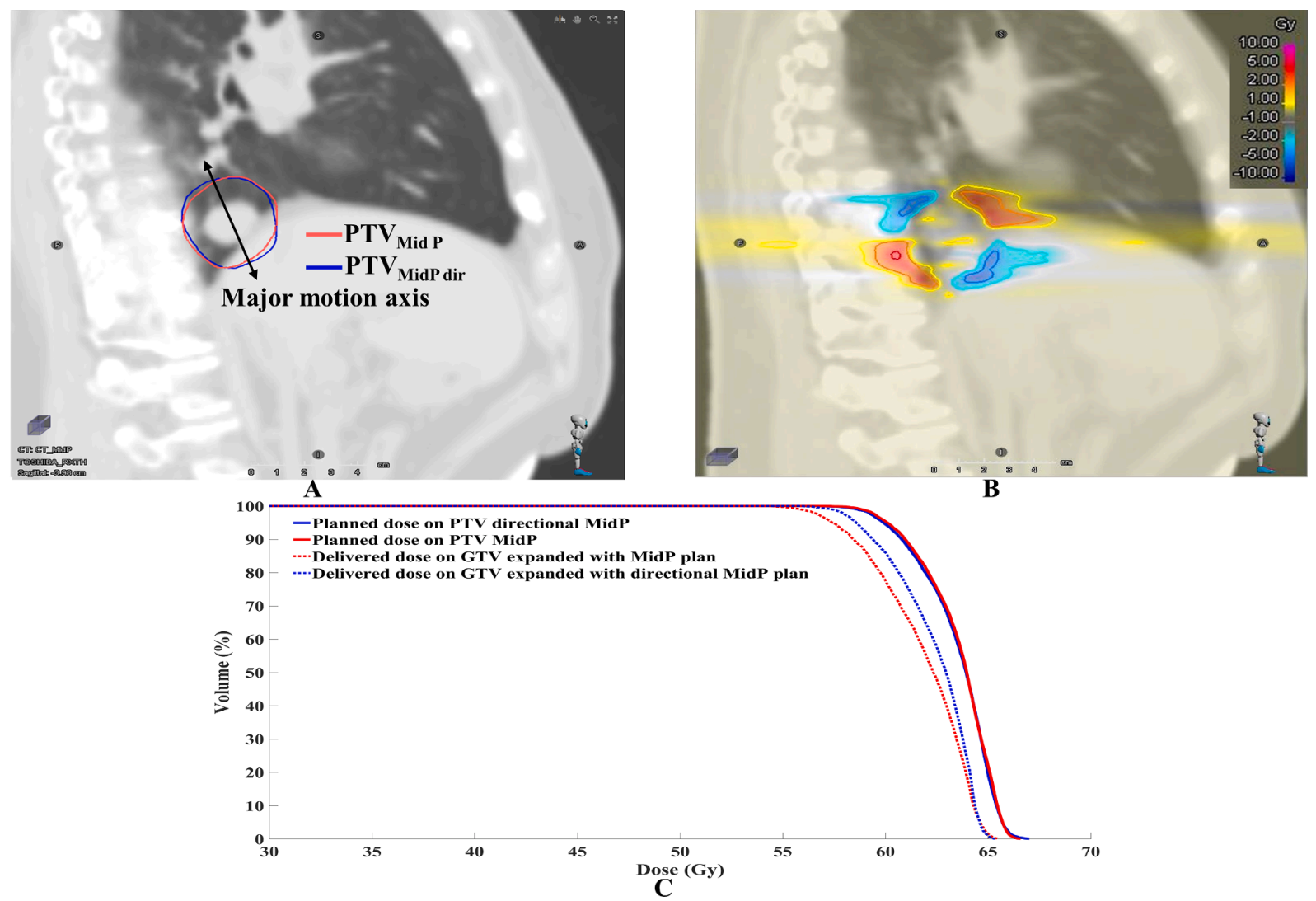

Fig. 4. A. MidP CT from lesion $\mathrm{n}^{\circ} 8$ with $\mathrm{PTV}_{\text {MidP }}$ and $\mathrm{PTV}_{\text {MidPdir. }}$ Black arrow indicates main motion direction in the sagittal plane. B. Differential dose map between MidP and directional MidP (negative values correspond to an overdosage with directional MidP) on the Average CT of lesion n ${ }^{\circ}$. C. Dose volume histogram showing planned doses to PTV and delivered dose to extended GTV for both methods.

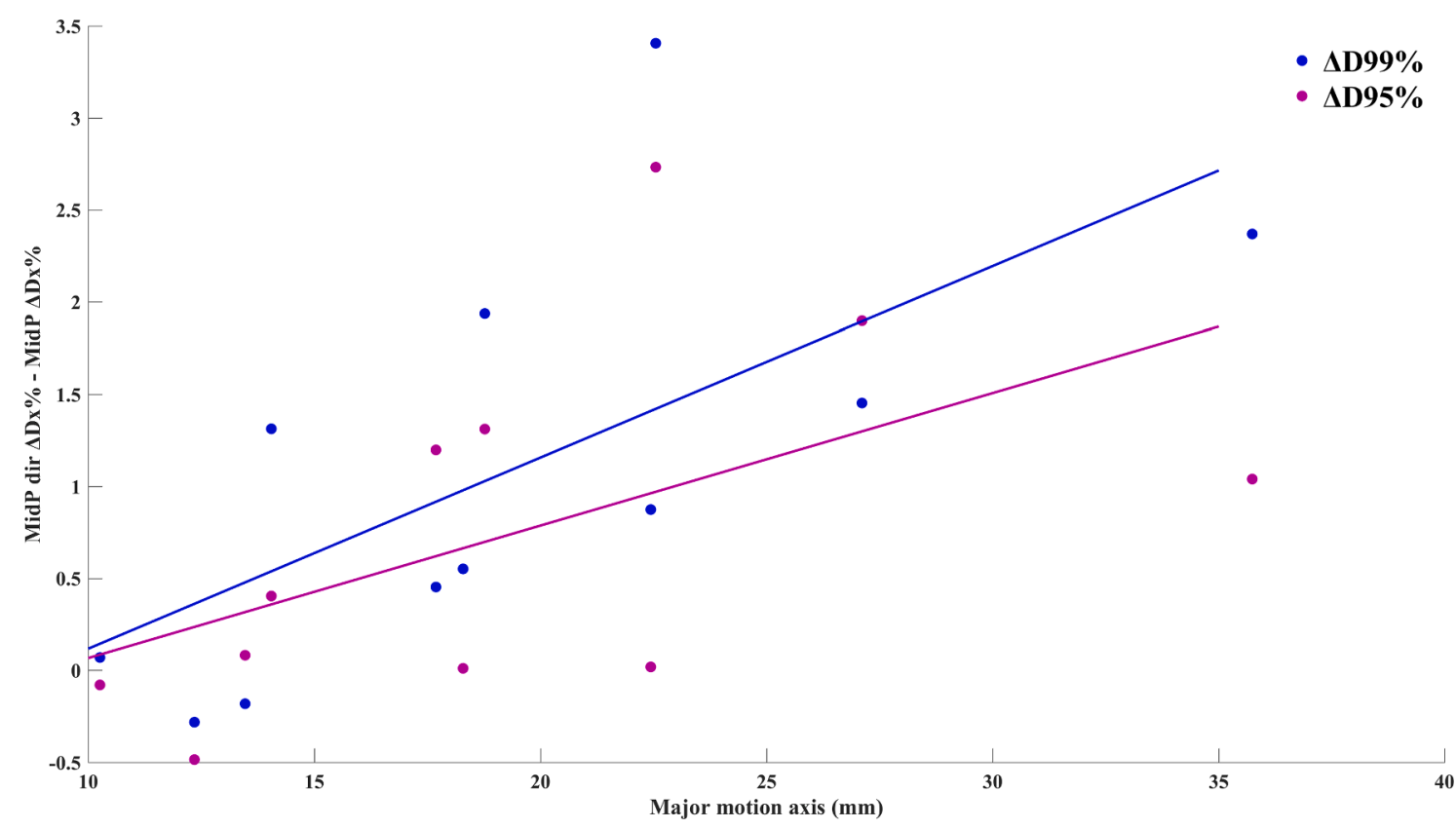

Fig. 5. Difference between MidP and directional MidP approach for $\Delta D_{95 \%}$ and $\Delta D_{99 \%}$ as a function of major motion axis amplitude.

variability. As illustrated in Fig. 5, it seems that dosimetric gain of PTV $_{\text {MidP dir }}$ is proportional to the motion amplitude. Tumor motion being the only error treated differently by the proposed method, this may be due to its larger weight with regard to the other geometrical uncertainties. It could thus be conjectured that the dosimetric advantage of directional MidP margins becomes also apparent for motions of lower amplitudes in case of larger coefficient $\beta$ e.g., normofractionated treatment of lung tumors [9], smaller penumbra e.g., liver SBRT [39] or less significant set-up [40] and systematic uncertainties [41-42]. Nevertheless, tumors with similar amplitudes can benefit very differently from 
Table 3

$\Delta \mathrm{D}_{99 \%}, \Delta \mathrm{D}_{95 \%}$ and $\Delta \mathrm{D}_{2 \%}$ for MidP and directional MidP methods.

\begin{tabular}{|c|c|c|c|c|c|c|}
\hline \multirow[t]{2}{*}{ Lesion } & \multicolumn{2}{|l|}{$\Delta D 99 \%$} & \multicolumn{2}{|l|}{$\Delta D 95 \%$} & \multicolumn{2}{|l|}{$\Delta \mathrm{D} 2 \%$} \\
\hline & MidP & MidP $_{\text {dir }}$ & MidP & $\mathbf{M i d P}_{\mathrm{dir}}$ & MidP & MidP $_{\text {dir }}$ \\
\hline 1 & $-2.96 \%$ & $-2.51 \%$ & $-2.42 \%$ & $-1.23 \%$ & $-2.02 \%$ & $-1.71 \%$ \\
\hline 2 & $-5.31 \%$ & $-3.99 \%$ & $-4.12 \%$ & $-3.72 \%$ & $-1.70 \%$ & $-1.97 \%$ \\
\hline 3 & $-6.61 \%$ & $-6.06 \%$ & $-4.93 \%$ & $-4.92 \%$ & $-1.45 \%$ & $-0.69 \%$ \\
\hline 4 & $-3.39 \%$ & $-3.57 \%$ & $-2.78 \%$ & $-2.70 \%$ & $-0.93 \%$ & $-0.50 \%$ \\
\hline 5 & $-5.02 \%$ & $-4.15 \%$ & $-4.36 \%$ & $-4.34 \%$ & $+2.58 \%$ & $+2.74 \%$ \\
\hline 6 & $-2.64 \%$ & $-2.93 \%$ & $-1.85 \%$ & $-2.33 \%$ & $-1.50 \%$ & $-1.90 \%$ \\
\hline 7 & $-1.50 \%$ & $-1.42 \%$ & $-0.53 \%$ & $-0.61 \%$ & $-0.44 \%$ & $-0.67 \%$ \\
\hline 8 & $-5.88 \%$ & $-2.48 \%$ & $-4.68 \%$ & $-1.95 \%$ & $-1.40 \%$ & $-1.78 \%$ \\
\hline 9 & $-7.31 \%$ & $-4.94 \%$ & $-6.23 \%$ & $-5.19 \%$ & $-0.11 \%$ & $-0.19 \%$ \\
\hline 10 & $-13.35 \%$ & $-11.89 \%$ & $-10.34 \%$ & $-8.54 \%$ & $-0.09 \%$ & $-0.05 \%$ \\
\hline 11 & $-10.48 \%$ & $-8.54 \%$ & $-7.23 \%$ & $-5.92 \%$ & $-0.08 \%$ & $-0.06 \%$ \\
\hline Mean & $-5.86 \%$ & $-4.77 \%$ & $-4.48 \%$ & $-3.74 \%$ & $-0.49 \%$ & $-0.40 \%$ \\
\hline p-value & $\mathrm{p}=0.011$ & & $\mathrm{p}=0.032$ & & $\mathrm{p}=0.429$ & \\
\hline
\end{tabular}
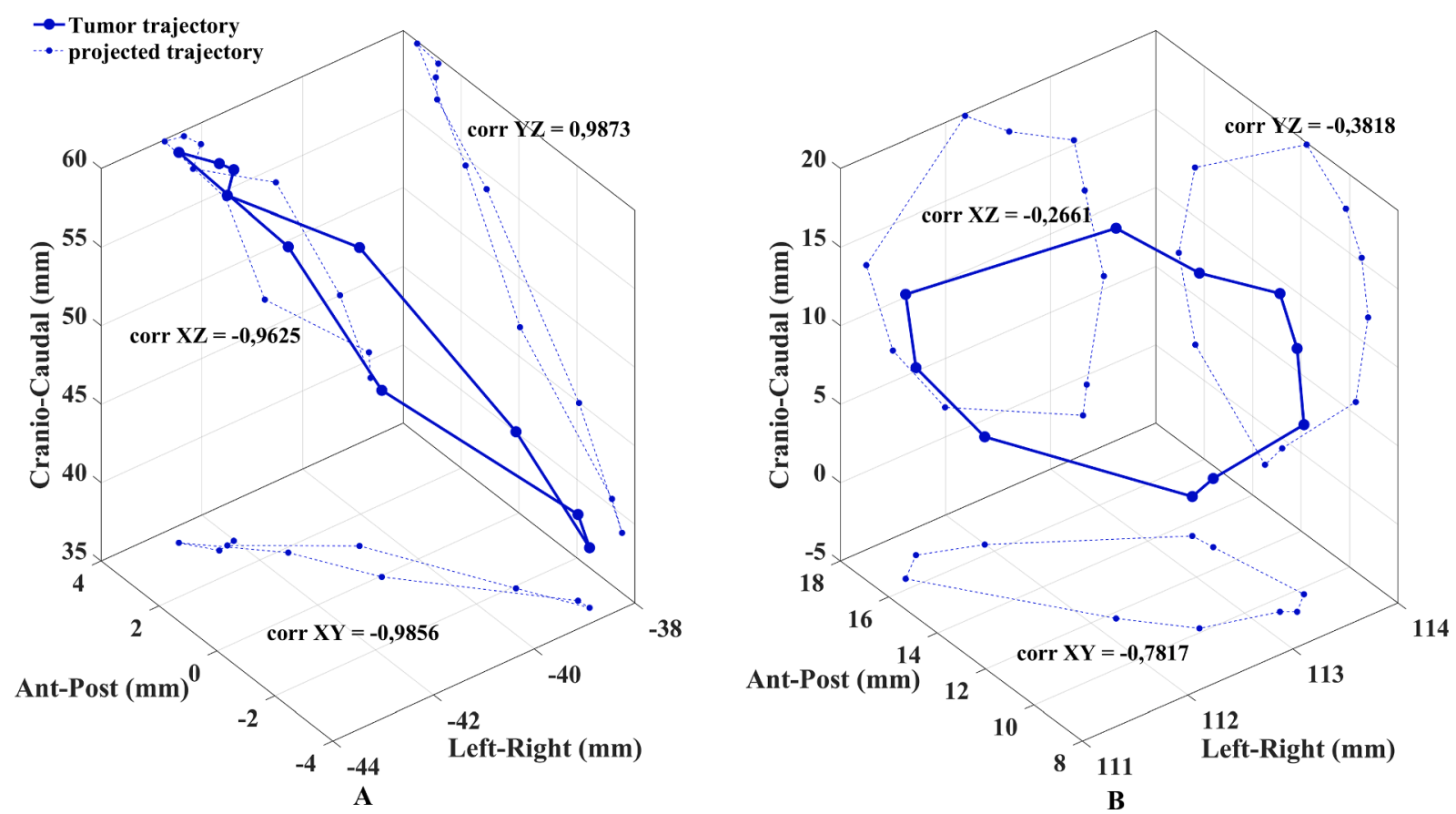

Fig. 6. Tumor trajectory and its projections with corresponding linear correlation coefficient for lesion $n^{\circ} 8$ (A) and lesion $n^{\circ} 5$ (B).

PTV MidP dir e.g., lesions $\mathrm{n}^{\circ} 8$ and 5 or lesions $\mathrm{n}^{\circ} 3$ and 11 . A first element could come from motion deviation from conventional axes. It is obvious to conclude that if principal motion axes are identical to the classical orthonormal axes, no difference will be observed between PTV $\mathrm{TidP}_{\text {and }}$ $\mathrm{PTV}_{\text {MidP dir. Lesions }} \mathrm{n}^{\circ} 8$ and 11 demonstrate indeed a larger motion obliquity compared to lesions $n^{\circ} 5$ and 3 . A second determinant could be related to tumor trajectory shape: van Herk's model assumes a null covariance between the different spatial components of geometric uncertainties. It means that LCC of tumor positions between conventional axes are also equal to zero. As illustrated in Fig. 6B, this approximation is quite acceptable in case of large hysteresis where tumor trajectory projected in the 3 spatial planes exhibits scatterplots with circular-like distributions characterized by a low LCC. However, for motions with linear morphology, this assumption is no longer realistic as represented in Fig. 6A. Motion amplitude being the same, it is therefore logical to

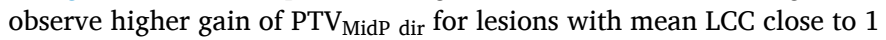
( ${ }^{\circ} 8$ and 11$)$ in comparison with tumors having lower mean LCC ( ${ }^{\circ} 3$ and 5). The influence of other motion pattern parameters on the absolute level of tumor dosimetric coverage highlighted by Wanet et al [14] such as motion amplitude or GTV size remain applicable. When motion amplitude increases or when random errors become non-negligible with respect to the GTV size, the model applicability is questionned. Some extreme cases can thus lead to inconsistent results regarding above described factors underlying PTV $_{\text {MidP dir }}$ benefit as for lesion 2, whose very small volume is of the order of $0.8 \mathrm{cc}$. Finally, 3 lesions with motion amplitude of less than $15 \mathrm{~mm}$ display a lower dosimetric coverage with directional margins (maximum difference of $0.49 \%$ for $\mathrm{D}_{95 \%}$ ). Apart from the fact that the clinical impact would be negligible, we attribute this small difference to the limit of similarity between PTV $_{\text {MidP }}$ and PTV $_{\text {MidP dir }}$ plan conformity for lesions where both approaches are most probably equivalent.

The patient preparation could affect the observed tumor motion, as well as its directionality. The impact of AC on tumor motion amplitude is well documented. It involves a significant reduction of motion amplitude for most of the liver and lung tumors [43]. A detrimental effect resulting in an increase of the motion amplitude could occur in $18 \%$ of the tumors [44]. However, little data is available regarding its influence on motion directionality. Worm et al [45] demonstrated that motion directionality was stable even when AC was applied. Indeed, AC was part of the treatment protocol for 5 of the 11 patients in their cohort. Unfortunately, the small sample size did not allow further conclusions to be drawn regarding its influence on the degree of motion obliquity. In 
our cohort, only 2 patients underwent AC. The same interpretative precautions as with the study of Worm et al [45] are necessary given the small sample size.

Margins generated by the directional MidP strategy consistently incorporate major motion axis as well as hysteresis amplitudes. This results in a wider PTV along the main motion direction but narrower perpendicularly. From a dosimetric point of view, it involves, as shown in Fig 4b, an overdosage at the extreme inspiratory and expiratory positions of breathing cycle corresponding to those where the tumor spends the most time. This explains the improved dose coverage achieved with PTV $_{\text {MidP dir. Directional margin validity will therefore depend }}$ on the stability of motion directionality and hysteresis. Fortunately, these two parameters are quite constant over time $[15,36]$. These metrics calculated on a 4D CT can thus be reliably extrapolated to the entire treatment. Nevertheless, the proposed method does not solve the important methodological weakness of margin strategy which consists in neglecting variability of tumor motion amplitude [46]. Regardless of the PTV computing method, margins strategies can be advantageously coupled with motion amplitude stabilizing approaches such as audio-video coaching [47] or more innovative techniques such as noninvasive mechanical ventilation using a portable artificial respirator widely available in hospital environment [48-49]. Motion amplitude variability could also be incorporated into the PTV margin but at the cost of a larger irradiation volume [50].

Alan McKenzie [15] has been indeed the first to propose aligning margins to the directions of tumor motion. Our method is similar to his as it is based on the decomposition of the covariance matrix of the geometric errors into eigenvalues. In contrast, motion uncertainty is treated differently. After the CTV expansion for systematic errors, a directional dilation involving only tumor motion is performed. This operation is identical to the proposition of Worm et al [45]. They advocated that the alignment of motion's margin should be made along the principal components of tumor motion. As part of of the directional MidP strategy, we have shown that the alignment of the directional margins only involves random uncertainties. It must also be performed along the principal components of the covariance matrix of the combined random errors and not those of the covariance matrix of tumor motion alone. The orthogonal basis defined by their eigenvectors is not the same indeed. Worm et al [45] also conjectured that the directional PTV volume would be smaller than the classical volume. In our study, the volumes obtained were similar between the investigated PTV definition methods.

In this work, directionality of baseline shift was not considered. Directional MidP method could be easily enhanced by assuming a nonnull covariances between dimensions of this geometrical uncertainty. Specification of its covariances would require a population-based quantification trough intra-fraction tumor positions monitoring. This is however out of the scope of this study.

Several limitations of this study need to be pointed out. The first concerns the use of a convolution collapsed cone algorithm for dosimetric analysis. Although an excellent agreement with Monte Carlo calculations is achieved in most cases, a slight overestimation of the dose at the target volume can be observed in some extreme conditions (small lung tumor) [51]. Since comparisons between MidP and directional MidP methods were performed on the same CTs for the same patients, it is reasonable to assume that this uncertainty has been propagated symmetrically between both approaches and that its net impact was negligible. Second, the 4D dose accumulation method used in this work did not consider the interplay effect. Residual incertitude related to tumor motion should be very small as it has been shown to be clinically insignificant [52-53]. In contrast, interplay effect induced by baseline shift could have a non-negligible dosimetric impact [54]. In the case of a large baseline shift, the study results should thus be extrapolated with caution. Lastly, rigid accumulation of the dose at the extended GTV did not take into account the dosimetric effects of tumor rotation.

Conclusions

The directional MidP method allows tumor motion to be better taken into account as a geometrical uncertainty without increasing the irradiation volume. This results in a higher dose delivered to the GTV than with the MidP approach, especially for highly mobile tumors whose trajectory has a linear shape and deviates from classical axes.

Fundings

Loïc Vander Veken is funded trough a FNRS grant FC $\mathrm{N}^{\circ} 33411$.

Kevin Souris is funded by the Walloon region (MECATECH/BIOWIN, grant number 8090).

\section{Declaration of Competing Interest}

The authors declare that they have no known competing financial interests or personal relationships that could have appeared to influence the work reported in this paper.

\section{Appendix n. 1}

Projected penumbra variances along axes of a new orthogonal basis ( $\left.x^{\prime}, y^{\prime}, z^{\prime}\right)$ can be written as follows :

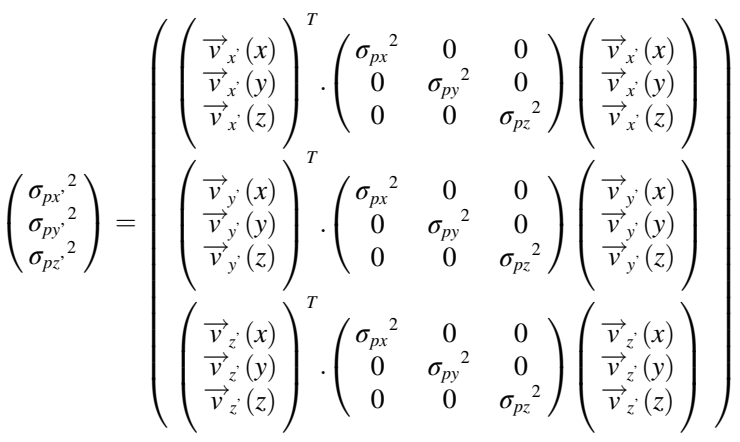

$$
\begin{aligned}
& \left(\begin{array}{c}
\sigma_{p x^{2}}{ }^{2} \\
\sigma_{p y^{\prime}}{ }^{2} \\
\sigma_{p z^{\prime}}{ }^{2}
\end{array}\right)=\left(\begin{array}{c}
\vec{v}_{x^{\prime}}(x)^{2} \cdot \sigma_{p x}{ }^{2}+\vec{v}_{x^{\prime}}(y)^{2} \cdot \sigma_{p y}{ }^{2}+\vec{v}_{x^{\prime}}(z)^{2} \cdot \sigma_{p z}{ }^{2} \\
\vec{v}_{y^{\prime}}(x)^{2} \cdot \sigma_{p x}{ }^{2}+\vec{v}_{y^{\prime}}(y)^{2} \cdot \sigma_{p y}{ }^{2}+\vec{v}_{y^{\prime}}(z)^{2} \cdot \sigma_{p z}{ }^{2} \\
\vec{v}_{z^{\prime}}(x)^{2} \cdot \sigma_{p x}{ }^{2}+\vec{v}_{z^{\prime}}(y)^{2} \cdot \sigma_{p y}{ }^{2}+\vec{v}_{z^{\prime}}(z)^{2} \cdot \sigma_{p z}{ }^{2}
\end{array}\right)
\end{aligned}
$$


$\left(\begin{array}{c}\sigma_{p x^{\prime}}{ }^{2} \\ \sigma_{p y^{\prime}}{ }^{2} \\ \sigma_{p z^{\prime}}{ }^{2}\end{array}\right)=\left(\begin{array}{l}\sigma_{p x}{ }^{2} \cdot\left(\vec{v}_{x^{\prime}}(x)^{2}+\vec{v}_{x^{\prime}}(y)^{2}+\vec{v}_{x^{\prime}}(z)^{2}\right) \\ \sigma_{p y}{ }^{2} \cdot\left(\vec{v}_{y}(x)^{2}+\vec{v}_{y^{\prime}}(y)^{2}+\vec{v}_{y^{\prime}}(z)^{2}\right) \\ \sigma_{p z}{ }^{2} \cdot\left(\vec{v}_{z^{\prime}}(x)^{2}+\vec{v}_{z^{\prime}}(y)^{2}+\vec{v}_{z^{\prime}}(z)^{2}\right)\end{array}\right)$

$\left(\begin{array}{c}\sigma_{p x^{\prime}}{ }^{2} \\ \sigma_{p y}{ }^{2} \\ \sigma_{p z^{\prime}}{ }^{2}\end{array}\right)=\left(\begin{array}{l}\sigma_{p x}{ }^{2} \\ \sigma_{p y}{ }^{2} \\ \sigma_{p z}{ }^{2}\end{array}\right)$

\section{References}

[1] Korreman SS. Motion in radiotherapy : photon therapy. Phys Med Biol 2012;57 (23):R161-91. https://doi.org/10.1088/0031-9155/57/23/r161.

[2] Anastasi G, Bertholet J, Poulsen P, Roggen T, Garibaldi C, Tilly N, et al. Patterns of practice for adaptive and real-time radiation therapy (POP-ART RT) part I : Intrafraction breathing motion management. Radiother Oncol 2020;2(3):1-2. https:// doi.org/10.1016/j.radonc.2020.06.018.

[3] Wolthaus JWH, Sonke J-J, van Herk M, Belderbos JSA, Rossi MMG, Lebesque JV, et al. Comparison of Different Strategies to Use Four-Dimensional Computed Tomography in Treatment Planning for Lung Cancer Patients. Int J Radiat Oncol*Biol*Phys 2008;70(4):1229-38. https://doi.org/10.1016/j. ijrobp.2007.11.042.

[4] Mutaf YD, Brinkmann DH. Optimization of Internal Margin to Account for Dosimetric Effects of Respiratory Motion. Int J Radiat Oncol*Biol*Phys 2008;70 (5):1561-70. https://doi.org/10.1016/j.ijrobp.2007.12.025.

[5] van Herk M, Remeijer P, Rasch C, Lebesque JV. The probability of correct target dosage: dose-population histograms for deriving treatment margins in radiotherapy. Int J Radiat Oncol*Biol*Phys 2000;47(4):1121-35. https://doi.org/ 10.1016/s0360-3016(00)00518-6.

[6] Wolthaus JWH, Schneider C, Sonke J-J, van Herk M, Belderbos JSA, Rossi MMG et al. Mid-ventilation CT scan construction from four-dimensional respirationcorrelated CT scans for radiotherapy planning of lung cancer patients. Int J Radiat Oncol*Biol*Phys 2006;65(5):1560-71. https://doi.org/10.1016/j. ijrobp.2006.04.031.

[7] Wolthaus JWH, Sonke J-J, van Herk M, Damen EMF. Reconstruction of a timeaveraged midposition CT scan for radiotherapy planning of lung cancer patients using deformable registrationa). Med Phys 2008;35(9):3998-4011. https://doi. org/10.1118/1.2966347.

[8] Jin P, Machiels M, Crama KF, Visser J, van Wieringen N, Bel A, et al. Dosimetric Benefits of Midposition Compared With Internal Target Volume Strategy for Esophageal Cancer Radiation Therapy. Int J Radiat Oncol*Biol*Phys 2019;103(2): 491-502. https://doi.org/10.1016/j.ijrobp.2018.09.024.

[9] Ayadi M, Baudier T, Bouilhol G, Dupuis P, Boissard P, Pinho R, et al. Mid-position treatment strategy for locally advanced lung cancer : a dosimetric study. Br J Radiol 2020;93(1110):20190692. https://doi.org/10.1259/bjr.20190692.

[10] Lens E, van der Horst A, Versteijne E, van Tienhoven G, Bel A. Dosimetric Advantages of Midventilation Compared With Internal Target Volume for Radiation Therapy of Pancreatic Cancer. Int J Radiat Oncol*Biol*Phys 2015;92(3): 675-82. https://doi.org/10.1016/j.ijrobp.2015.02.015.

[11] Ehrbar S, Perrin R, Peroni M, Bernatowicz K, Parkel T, Pytko I, et al. Respiratory motion-management in stereotactic body radiation therapy for lung cancer - A dosimetric comparison in an anthropomorphic lung phantom (LuCa). Radiother Oncol 2016;121(2):328-34. https://doi.org/10.1016/j.radonc.2016.10.011.

[12] Thomas SJ, Evans BJ, Harihar L, Chantler HJ, Martin AGR, Harden SV. An evaluation of the mid-ventilation method for the planning of stereotactic lung plans. Radiother Oncol 2019;137:110-6. https://doi.org/10.1016/j. radonc.2019.04.031.

[13] Arab-Ceschia F, Chajon E, Lafond C, Bellec J. 1 Comparison of target coverage for two motion-encompassing methods for lung stereotactic body radiotherapy by using in-treatment 4D cone-beam CT. Physica Med 2018;56:1. https://doi.org/ 10.1016/j.ejmp.2018.09.014.

[14] Wanet M, Sterpin E, Janssens G, Delor A, Lee JA, Geets X. Validation of the midposition strategy for lung tumors in helical TomoTherapy. Radiother Oncol 2014; 110(3):529-37. https://doi.org/10.1016/j.radonc.2013.10.025.

[15] McKenzie A. Appendix 2A General method for combining errors. In : British Institute of Radiology. Geometric Uncertainties in Radiotherapy. Br Instit Radiol; 2003: p. 28-34.

[16] McKenzie AL. How should breathing motion be combined with other errors when drawing margins around clinical target volumes ? Br J Radiol 2000;73(873):973-7. https://doi.org/10.1259/bjr.73.873.11064651.

[17] Janssens G. Registration models for tracking organs and tumors in highly deformable anatomies : applications to radiotherapy. UNIVERSITE CATHOLIQUE DE LOUVAIN 2010. http://hdl.handle.net/2078.1/33459.

[18] Lujan AE, Larsen EW, Balter JM, Ten Haken RK. A method for incorporating organ motion due to breathing into 3D dose calculations. Med Phys 1999;26(5):715-20. https://doi.org/10.1118/1.598577.

[19] Bissonnette J-P, Franks KN, Purdie TG, Moseley DJ, Sonke J-J, Jaffray DA, et al. Quantifying Interfraction and Intrafraction Tumor Motion in Lung Stereotactic Body Radiotherapy Using Respiration-Correlated Cone Beam Computed
Tomography. Int J Radiat Oncol*Biol*Phys 2009;75(3):688-95. https://doi.org/ 10.1016/j.ijrobp.2008.11.066.

[20] Sonke J-J, Lebesque J, van Herk M. Variability of Four-Dimensional Computed Tomography Patient Models. Int J Radiat Oncol*Biol*Phys 2008;70(2):590-8. https://doi.org/10.1016/j.ijrobp.2007.08.067.

[21] Witte MG, van der Geer J, Schneider C, Lebesque JV, van Herk M. The effects of target size and tissue density on the minimum margin required for random errors. Med Phys 2004;31(11):3068-79. https://doi.org/10.1118/1.1809991.

[22] Han K, Basran PS, Cheung P. Comparison of Helical and Average Computed Tomography for Stereotactic Body Radiation Treatment Planning and Normal Tissue Contouring in Lung Cancer. Clin Oncol 2010;22(10):862-7. https://doi.org/ 10.1016/j.clon.2010.08.010.

[23] Guckenberger M, Andratschke N, Dieckmann K, Hoogeman MS, Hoyer M, Hurkmans C, et al. ESTRO ACROP consensus guideline on implementation and practice of stereotactic body radiotherapy for peripherally located early stage nonsmall cell lung cancer. Radiother Oncol 2017;124(1):11-7. https://doi.org/ 10.1016/j.radonc.2017.05.012.

[24] Xiao Y, Papiez L, Paulus R, Timmerman R, Straube WL, Bosch WR, et al. Dosimetric Evaluation of Heterogeneity Corrections for RTOG 0236: Stereotactic Body Radiotherapy of Inoperable Stage I-II Non-Small-Cell Lung Cancer. Int J Radiat Oncol*Biol*Phys 2009;73(4):1235-42. https://doi.org/10.1016/j. ijrobp.2008.11.019.

[25] Timmerman R, Galvin J, Michalski J, Straube W, Ibbott G, Martin E, et al. Accreditation and quality assurance for Radiation Therapy Oncology Group : Multicenter clinical trials using Stereotactic Body Radiation Therapy in lung cancer. Acta Oncol 2006;45(7):779-86. https://doi.org/10.1080/ 02841860600902213.

[26] Paddick I. A simple scoring ratio to index the conformity of radiosurgical treatment plans. J Neurosurg 2000;93(supplement_3):219-22. https://doi.org/10.3171/ jns.2000.93.supplement 3.0219.

[27] Feuvret L, Noël G, Mazeron JJ, Bey P. Conformity index : A review. Int J Radiat Oncol*Biol*Phys 2006;64(2):333-42. https://doi.org/10.1016/j. ijrobp.2005.09.028.

[28] Dice LR. Measures of the Amount of Ecologic Association Between Species. Ecology 1945;26(3):297-302. https://doi.org/10.2307/1932409.

[29] Shintani, T., Nakamura, M., Matsuo, Y., Miyabe, Y., Mukumoto, N., Mitsuyoshi, T., Iizuka, Y., \& Mizowaki, T. Investigation of 4D dose in volumetric modulated arc therapy-based stereotactic body radiation therapy : does fractional dose or number of arcs matter? J Radiat Res, 61(2); 2020a. 325-334. 10.1093/jrr/rrz103.

[30] Studenski MT, Delgadillo R, Xu Y, Both J, Padgett K, Abramowitz M, et al. Margin verification for hypofractionated prostate radiotherapy using a novel dose accumulation workflow and iterative CBCT. Physica Med 2020;77:154-9. https:// doi.org/10.1016/j.ejmp.2020.08.008.

[31] Leung RWK, Chan MKH, Chiang C-L, Wong M, Blanck O. On the pitfalls of PTV in lung SBRT using type-B dose engine : an analysis of PTV and worst case scenario concepts for treatment plan optimization. Radiat Oncol 2020;15(1). https://doi. org/10.1186/s13014-020-01573-9.

[32] Glide-Hurst CK, Hugo GD, Liang J, Yan D. A simplified method of four-dimensional dose accumulation using the mean patient density representation. Med Phys 2008; 35(12):5269-77. https://doi.org/10.1118/1.3002304.

[33] Murrell DH, Laba JM, Erickson A, Millman B, Palma DA, Louie AV. Stereotactic ablative radiotherapy for ultra-central lung tumors : prioritize target coverage or organs at risk ? Radiat Oncol 2018;13(1). https://doi.org/10.1186/s13014-0181001-6.

[34] Loi M, Franceschini D, Dominici L, Chiola I, Franzese C, D'Agostino GR, et al. Dose coverage impacts local control in ultra-central lung oligometastases treated with stereotactic radiotherapy. Strahlenther Onkol 2020;(1). https://doi.org/10.1007/ s00066-020-01687-9.

[35] Swaminath A, Massey C, Brierley JD, Dinniwell R, Wong R, Kim JJ, et al. Accumulated Delivered Dose Response of Stereotactic Body Radiation Therapy for Liver Metastases. Int J Radiat Oncol*Biol*Phys 2015;93(3):639-48. https://doi. org/10.1016/j.ijrobp.2015.07.2273.

[36] Karlsson K, Lax I, Lindbäck E, Grozman V, Lindberg K, Wersäll P, et al. Estimation of delivered dose to lung tumours considering setup uncertainties and breathing motion in a cohort of patients treated with stereotactic body radiation therapy. Physica Med 2021;88:53-64. https://doi.org/10.1016/j.ejmp.2021.06.015.

[37] Guckenberger M, Klement RJ, Allgäuer M, Andratschke N, Blanck O, BodaHeggemann J, et al. Local tumor control probability modeling of primary and secondary lung tumors in stereotactic body radiotherapy. Radiother Oncol 2016; 118(3):485-91. https://doi.org/10.1016/j.radonc.2015.09.008. 
[38] van Baardwijk A, Tomé WA, van Elmpt W, Bentzen SM, Reymen B, Wanders R, et al. Is high-dose stereotactic body radiotherapy (SBRT) for stage I non-small cell lung cancer (NSCLC) overkill ? A systematic review. Radiotherapy and Oncology 2012;105(2):145-9. https://doi.org/10.1016/j.radonc.2012.09.008.

[39] Velec M, Moseley JL, Dawson LA, Brock KK. Dose Escalated Liver Stereotactic Body Radiation Therapy at the Mean Respiratory Position. Int J Radiat Oncol*Biol*Phys 2014;89(5):1121-8. https://doi.org/10.1016/j.ijrobp.2014.04.051.

[40] Vloet A, Li W, Giuliani M, Seco P, Silver L, Sun A, et al. Comparison of residual geometric errors obtained for lung SBRT under static beams and VMAT techniques : Implications for PTV margins. Physica Med 2018;52:129-32. https://doi.org/ 10.1016/j.ejmp.2018.07.009.

[41] Dechambre D, Vander Veken L, Delor A, Sterpin E, Vanneste F, Geets X. Feasibility of a TPS-integrated method to incorporate tumor motion in the margin recipe. Med Dosim Published. 2021;46(3):253-8. https://doi.org/10.1016/j. meddos.2021.02.002.

[42] Bernstein D, Taylor A, Nill S, Oelfke U. New target volume delineation and PTV strategies to further personalise radiotherapy. Phys Med Biol 2021;66(5):055024. https://doi.org/10.1088/1361-6560/abe029.

[43] Eccles CL, Patel R, Simeonov AK, Lockwood G, Haider M, Dawson LA. Comparison of Liver Tumor Motion With and Without Abdominal Compression Using CineMagnetic Resonance Imaging. Int J Radiat Oncol*Biol*Phys 2011;79(2):602-8. https://doi.org/10.1016/j.ijrobp.2010.04.028.

[44] Bouilhol G, Ayadi M, Rit S, Thengumpallil S, Schaerer J, Vandemeulebroucke J, et al. Is abdominal compression useful in lung stereotactic body radiation therapy? A 4DCT and dosimetric lobe-dependent study. Physica Med 2013;29(4):333-40. https://doi.org/10.1016/j.ejmp.2012.04.006.

[45] Worm ES, Høyer M, Fledelius W, Hansen AT, Poulsen PR. Variations in magnitude and directionality of respiratory target motion throughout full treatment courses of stereotactic body radiotherapy for tumors in the liver. Acta Oncol 2013;52(7): 1437-44. https://doi.org/10.3109/0284186x.2013.813638.

[46] Dhont J, Vandemeulebroucke J, Burghelea M, Poels K, Depuydt T, Van Den Begin R, et al. The long- and short-term variability of breathing induced tumor motion in lung and liver over the course of a radiotherapy treatment. Radiother Oncol 2018;126(2):339-46. https://doi.org/10.1016/j.radonc.2017.09.001.
[47] Goossens S, Senny F, Lee JA, Janssens G, Geets X. Assessment of tumor motion reproducibility with audio-visual coaching through successive 4D CT sessions. J Appl Clin Med Phys 2014;15(1):47-56. https://doi.org/10.1120/jacmp. v15i1.4332.

[48] Van Ooteghem G, Dasnoy-Sumell D, Lambrecht M, Reychler G, Liistro G, Sterpin E, et al. Mechanically-assisted non-invasive ventilation : A step forward to modulate and to improve the reproducibility of breathing-related motion in radiation therapy. Radiother Oncol 2019;133:132-9. https://doi.org/10.1016/j. radonc.2018.12.026.

[49] Van Ooteghem G, Dasnoy-Sumell D, Lee JA, Geets X. Mechanically-assisted and non-invasive ventilation for radiation therapy : A safe technique to regularize and modulate internal tumour motion. Radiother Oncol 2019;141:283-91. https://doi. org/10.1016/j.radonc.2019.09.021.

[50] Sevillano D, Núñez L, Chevalier M, García-Vicente F. Application of discrete cosine transform to assess the effect of tumor motion variations on the definition of ITV in lung and liver SBRT. Physica Med 2021;84:132-40. https://doi.org/10.1016/j. ejmp.2021.03.036.

[51] Sterpin E, Salvat F, Olivera G, Vynckier S. Monte Carlo evaluation of the convolution/superposition algorithm of $\mathrm{Hi}-\mathrm{Art}^{\mathrm{TM}}$ tomotherapy in heterogeneous phantoms and clinical cases. Med Phys 2009;36(5):1566-75. https://doi.org/ $10.1118 / 1.3112364$.

[52] Sterpin E, Janssens G, Orban de Xivry J, Goossens S, Wanet M, Lee JA, et al. Helical tomotherapy for SIB and hypo-fractionated treatments in lung carcinomas : A 4D Monte Carlo treatment planning study. Radiother Oncol 2012;104(2):173-80. https://doi.org/10.1016/j.radonc.2012.06.005.

[53] Rao M, Wu J, Cao D, Wong T, Mehta V, Shepard D, et al. Dosimetric Impact of Breathing Motion in Lung Stereotactic Body Radiotherapy Treatment Using ImageModulated Radiotherapy and Volumetric Modulated Arc Therapy. Int J Radiat Oncol*Biol*Phys 2012;83(2):e251-6. https://doi.org/10.1016/j. ijrobp.2011.12.001.

[54] Tudor GSJ, Harden SV, Thomas SJ. Three-dimensional analysis of the respiratory interplay effect in helical tomotherapy : Baseline variations cause the greater part of dose inhomogeneities seen. Med Phys 2014;41(3):031704. https://doi.org/ $10.1118 / 1.4864241$. 